\title{
Hybrid (2D/3D) Dosimetry of Radiolabeled Gold Nanoparticles for Sentinel Lymph Node Detection in Patients with Breast Cancer
}

\author{
Gerardo Ramírez-Nava (iD, ${ }^{1,2}$ Clara Santos-Cuevas ${ }^{(D},{ }^{1}$ Guillermina Ferro-Flores ${ }^{D}{ }^{1}$ \\ Blanca Ocampo-García, ${ }^{1}$ Isaac Chairez, ${ }^{2}$ Edgar Gómez-Argumosa, ${ }^{3}$ \\ Lucero Abundiz-López, ${ }^{3}$ and Francisco O. García-Pérez ${ }^{3}$ \\ ${ }^{1}$ Departamento de Materiales Radiactivos, Instituto Nacional de Investigaciones Nucleares, Ocoyoacac 52750, \\ Estado de México, Mexico \\ ${ }^{2}$ Departamento de Bioprocesos, Instituto Politécnico Nacional, Ciudad de México 07340, Mexico \\ ${ }^{3}$ Departamento de Medicina Nuclear, Instituto Nacional de Cancerología, Ciudad de México 14000, Mexico
}

Correspondence should be addressed to Clara Santos-Cuevas; clara.cuevas@inin.gob.mx and Guillermina Ferro-Flores; ferro_flores@yahoo.com.mx

Received 27 September 2019; Accepted 28 December 2019; Published 7 May 2020

Academic Editor: Fernando Herranz

Copyright (C) 2020 Gerardo Ramírez-Nava et al. This is an open access article distributed under the Creative Commons Attribution License, which permits unrestricted use, distribution, and reproduction in any medium, provided the original work is properly cited.

\begin{abstract}
Previously, we reported the preparation and preclinical studies of ${ }^{99 \mathrm{~m}} \mathrm{Tc}$-labeled gold nanoparticles-mannose $\left({ }^{99 \mathrm{~m}} \mathrm{Tc}\right.$-AuNPmannose) with potential for sentinel lymph node (SLN) detection by using nuclear medicine procedures. This study aimed to evaluate the biokinetics and hybrid (2D/3D) dosimetry of ${ }^{99 \mathrm{~m}} \mathrm{Tc}$-AuNP-mannose in five patients with breast cancer under a sentinel lymph node detection protocol. Anterior and posterior whole-body planar images (2D, at $0.5,2,6$, and $24 \mathrm{~h})$ and singlephoton emission computed tomography (3D at $6.5 \mathrm{~h}$ )/computed tomography (SPECT/CT) images were acquired after ${ }^{99 \mathrm{~m}} \mathrm{Tc}-$ AuNP-mannose administration (37 MBq). Through a hybrid quantification method, activity in tissues of interest at the different acquisition times was determined and integrated over time to obtain the total nuclear transformations $(N)$, as well as the mean residence time, in each tissue. $N$ values and the OLINDA code were used for estimating the internal radiation absorbed doses. Results demonstrated that ${ }^{99 \mathrm{~m}} \mathrm{Tc}$-AuNP-mannose successfully accumulates and remains up to $24 \mathrm{~h}$ in the sentinel lymph node without detectable migration to other lymph nodes and no side effects on patients. Negligible absorption of the radiolabeled nanoparticles into the circulatory system was observed, from which the radio-nanosystem is rapidly eliminated by kidneys. Hybrid $(2 \mathrm{D} / 3 \mathrm{D})$ dosimetry evaluations showed equivalent doses to SLN, breast, and kidneys of $172.34,5.32$, and $0.08 \mathrm{mSv} / 37 \mathrm{MBq}$, respectively, with an effective dose of $2.05 E-03 \mathrm{mSv} / \mathrm{MBq}$. The mean effective residence time in SLN was $0.92 \mathrm{~h}$. This preliminary study indicates that the use of ${ }^{99 \mathrm{~m}} \mathrm{Tc}$-AuNP-mannose for successful SLN detection in patients is safe, producing an effective dose at the level recommended for diagnostic studies $(<10 \mathrm{mSv})$.
\end{abstract}

\section{Introduction}

In breast cancer patients, the sentinel lymph node (SLN) is defined as the first lymph node that malignant cells reach when migrating from the primary tumor $[1,2]$. The histological study of the SLN for evaluation of cancer cell invasiveness is crucial in disease prognosis. For SLN detection, a blue dye or a colloid radiopharmaceutical, or both, are usually employed. Techniques for SLN detection improve the accuracy of surgical and biopsy procedures $[2,3]$.
However, dyes or radiopharmaceuticals currently available for clinical use are released from the SLN to other lymph nodes in a relatively short time [3].

The developments on receptor-specific/biocompatible nanoparticles $(1-100 \mathrm{~nm})$, useful as diagnostic, therapeutic, and drug delivery systems, have demonstrated the potential of nanotechnology in the field of biomedical imaging and medicine $[4,5]$. Among others, gold nanoparticles (AuNPs) have suitable properties for many biomedical applications [6]. Recently, different systems based on AuNPs have been 
developed and preclinically assessed for SLN detection $[5,7,8]$. In our case, ${ }^{99 \mathrm{~m}} \mathrm{Tc}$-labeled AuNP-mannose $\left({ }^{99 \mathrm{~m}} \mathrm{Tc}\right.$ AuNP-mannose) was prepared as a radiotracer to specifically target mannose receptors of macrophages abundantly present in the SLN [5]. Preclinical studies demonstrated that ${ }^{99} \mathrm{~m}$ Tc-AuNP-mannose is significantly retained in the first lymph node of Wistar rats from $1 \mathrm{~h}$ to at least $24 \mathrm{~h}$ after intradermal administration. Due to these characteristics, ${ }^{99 \mathrm{~m}}$ Tc-AuNP-mannose could be used for SLN detection using 1-day or 2-day clinical protocols [9].

Although planar lymphoscintigraphy has been widely used for SLN detection $[9,10]$, the single-photon emission computed tomography (SPECT) 3D imaging, coupled with computed tomography (CT), has improved the identification of SLN in breast cancer patients [10-12]. Quantitative 3D SPECT/CT imaging is the most accurate method for evaluating dosimetry in patients; however, multiple 3D images must be acquired at numerous time points, resulting in prolonged and uncomfortable sessions for patients. Recently, hybrid planar/SPECT (2D/3D) quantification methods have been proposed to obtain biokinetic and dosimetric data of radiopharmaceuticals in a relatively short time $[13,14]$. These methods employ multiple planar images to get the biodistribution models and at least one SPECT/CT image to scale the models of organs and tissues of interest to 3D by using specific imaging correction factors [13-15].

This study addresses the biokinetics and hybrid (2D/3D) dosimetry of ${ }^{99 \mathrm{~m}} \mathrm{Tc}$-AuNP-mannose in five patients with breast cancer under an SLN detection protocol.

\section{Materials and Methods}

2.1. Preparation of ${ }^{99 m}$ Tc-AuNP-Mannose. Technetium-99m-labeled AuNP-mannose was obtained by adding ${ }^{99 \mathrm{~m}} \mathrm{Tc}$-EDDA/HYNIC-Tyr ${ }^{3}$-Octreotide $\quad(0.1 \mathrm{~mL}$; $0.15 \mathrm{GBq} ; 0.3 \mu$ moles of peptide; $1.3 \mathrm{E} 14$ molecules) to a sterile solution of AuNP-mannose $(1.5 \mathrm{~mL} ; 12 \mathrm{~nm} ; 6 \mathrm{E} 12$ particles) prepared in a GMP-certified facility, as previously reported [16]. Radiochemical purity of $>95 \%$ was corroborated by ITLC-SG/methyl-ethyl-ketone $\left(R_{\mathrm{f}}=0.0\right.$ for ${ }^{99 \mathrm{~m}} \mathrm{Tc}$ AuNP-mannose and $R_{\mathrm{f}}=1.0$ for $\left.{ }^{99} \mathrm{~m} \mathrm{TcO}_{4} \mathrm{Na}\right)$ and ultrafiltration (Amicon Ultracel, Millipore, 30,000 MW cutoff), in which ${ }^{99 \mathrm{~m}} \mathrm{Tc}$-AuNP-mannose remains in the filter, whereas ${ }^{99 \mathrm{~m}} \mathrm{Tc}$-EDDA/HYNIC-Tyr ${ }^{3}$-octreotide and ${ }^{99 \mathrm{~m} \mathrm{TcO}_{4} \mathrm{Na} \text { pass }}$ through the filter.

\subsection{Clinical Studies}

2.2.1. Patients. After being approved by the hospital's Medical Ethics Committee, the study enrolled five female patients (Table 1), diagnosed with breast cancer (mean age $\pm S D, 53.60 \pm 19.54$ y; age range: $29-76$ ). All patients received detailed information about the procedures and the aim of the study. Everyone agreed to participate and signed a consent form. The activity administered to each patient was $37 \mathrm{MBq}$, divided into four equal aliquots, which were injected using the intradermal periareolar technique.
TABLE 1: Patients with breast cancer included in the SLN detection protocol with ${ }^{99 \mathrm{~m}} \mathrm{Tc}$-AuNP-mannose.

\begin{tabular}{lcc}
\hline Patient no. & Age & Disease \\
\hline 1 & 47 & Ductal carcinoma in situ \\
2 & 29 & Ductal carcinoma in situ (T1a*) \\
3 & 71 & Ductal carcinoma in situ (T1b*) \\
4 & 76 & Ductal carcinoma in situ \\
5 & 45 & Ductal carcinoma in situ (Tla*) \\
\hline
\end{tabular}

${ }^{*}$ According to the TNM classification of malignant tumors [17].

2.2.2. Imaging Studies. ${ }^{99 \mathrm{~m}} \mathrm{Tc}-\mathrm{AuNP}$-mannose planar and SPECT/CT images were obtained to calculate the biokinetic and dosimetry parameters with a dual-head gamma camera (Symbia TruePoint SPECT/CT, Siemens), equipped with low-energy high-resolution (LEHR) collimators.

(1) Planar Imaging. The photopeak window was centered at $140 \mathrm{keV}$ with a width of $15 \%(129.5-150.5 \mathrm{keV})$. To correct the photon scattering using a dual-energy window method, a lower window centered at $119 \mathrm{keV}$ and a $15 \%$ width (108.5-129.5 keV), was set. The scan velocity was $12 \mathrm{~cm} / \mathrm{min}$, and the size of the matrix was $256 \times 1024$ pixels.

The chest and abdomen transmission factors were calculated using the ratio of the count rates $I_{\mathrm{P}} / I_{\mathrm{WP}}$, obtained with a $37 \mathrm{MBq}{ }^{99 \mathrm{~m}}$ Tc-filled flood source, with $\left(I_{\mathrm{P}}\right)$ and without $\left(I_{\mathrm{WP}}\right)$ the patient, from which the regional attenuation of the body was calculated. Anterior and posterior scintigraphy of whole-body was performed at $0.5,2,6$, and $24 \mathrm{~h}$ after radiopharmaceutical administration $[18,19]$.

(2) SPECT/CT. The SPECT images were acquired using the same collimators and energy window configuration described in the previous section. Each study consisted of 120 projections covering $360^{\circ}$; the acquisition time of each projection was 15 seconds. The matrix size was set to $128 \times 128$ pixels, and the pixel size was set to $4.8 \mathrm{~mm}$. The reconstruction of the nuclear images was obtained through the Flash-3D algorithm (modified form of the OSEM algorithm), considering four subsets, eight iterations, and no smoothing filter. The CT images were obtained with $130 \mathrm{kV}$ and $30 \mathrm{mAs}$. The reconstruction algorithm used in these images was the filtered backprojection (FBP). The matrix size was set to $512 \times 512$ pixels, and pixel size was set to $1.2 \mathrm{~mm}$. The thicknesses of the reconstructed slices were 1.2 and $5 \mathrm{~mm}$. The CT reconstructions with slices of $1.2 \mathrm{~mm}$ were used to draw the regions of interest (ROIs), to obtain segmented volumes of interest (VOIs). The CT slices of $5 \mathrm{~mm}$ were used to get the attenuation map, in order to apply attenuation correction in the SPECT images. The SPECT/CT images of the chest and abdomen were performed $6.5 \mathrm{~h}$ after radiopharmaceutical administration [20, 21].

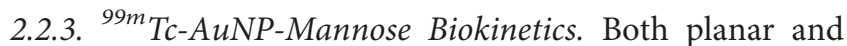
SPECT images were archived in the DICOM (Digital Imaging and Communication in Medicine) format and processed with Matlab (MathWorks, 2018), ImageJ (National Institute of Health, 2016) and OsiriX MD (Pixmeo, 2019). 
(1) Planar Imaging. The planar images were corrected by attenuation using the transmission factors $I_{\mathrm{P}} / I_{\mathrm{WP}}$. The scattering correction in these images was achieved with the method proposed by Koral et al. In this method, the true photopeak counts $T_{\mathrm{PC}}$ are given by the following equation [22]:

$$
T_{\mathrm{PC}}=C_{\mathrm{PK}}-m_{\mathrm{f}} C_{\mathrm{ST}},
$$

where $C_{\mathrm{PK}}$ is the total count recorded within the photopeak window, $C_{\mathrm{ST}}$ is the count within the scatter window, and $m_{\mathrm{f}}$ is a multiplying factor $\left(m_{\mathrm{f}}=0.5\right.$ is commonly used for ${ }^{99} \mathrm{~m}$ Tc). ROIs were drawn around source organs (mammary glands, SLN, kidneys, urinary bladder, and whole-body) in each time frame. For all scans, the same set of ROIs was used, and the counts in each ROI were corrected by attenuation using the transmission factors $\left(I_{\mathrm{P}} / I_{\mathrm{WP}}\right)$ experimentally calculated as previously mentioned, according to the conjugate-view counting method for additional scattering correction, as follows:

$$
A_{\mathrm{ROI}}=\frac{I_{\mathrm{P}}}{I_{\mathrm{WP}}} \sqrt{I_{\mathrm{ANT}} I_{\mathrm{POST}}}
$$

where $A_{\mathrm{ROI}}$ is the activity in the compartment understudy, $\left(I_{\mathrm{P}} / I_{\mathrm{WP}}\right)$ is the transmission factor experimentally calculated, and $I_{\mathrm{ANT}}$ and $I_{\mathrm{POST}}$ are the anterior and posterior counting rates, respectively. The counts were also corrected by physical decay. The activity of each organ was divided by the whole-body (WB) activity obtained from the first image acquired (100\% of injected activity). The fraction of the injected activity (INA) in each source organ was calculated as follows:

$$
\% \text { INA }=\frac{A_{\text {source organ }}}{A_{\text {WB at the first acquisition }}} \times 100 .
$$

(2) SPECT/CT. The SPECT images were corrected by attenuation with the attenuation maps, which were obtained using the conversion of $\mathrm{HU}$ to linear attenuation coefficients. The photon scattering was corrected with a dualenergy method, which employs a single lower scatter window adjacent to the photopeak window. The scatter estimate $S E_{P P}$ within the photopeak window is given by the following equation:

$$
\mathrm{SE}_{\mathrm{PP}}=\left(\frac{W_{\mathrm{PK}}}{2 W_{\mathrm{ST}_{1}}}\right)\left(P_{\mathrm{ST}_{1}}\right)
$$

where $W_{\mathrm{PK}}$ and $W_{\mathrm{ST}_{1}}$ are the photopeak window PK widths and the scatter window $\mathrm{ST}_{1}$, respectively. $P_{\mathrm{ST}_{1}}$ is the projection image within the scatter window $\mathrm{ST}_{1}$ [20].

The system sensitivity factor, $S_{\text {SPECT }}(\mathrm{cps} / \mathrm{MBq})$, was obtained with the following equation:

$$
S_{\mathrm{SPECT}}=\frac{\mathrm{CR}_{\mathrm{VOI}}\left(e\left(\left(T_{\mathrm{S}}-T_{\mathrm{CL}}\right) / T_{1 / 2}\right)\right)\left(T_{\mathrm{TAT}} \ln (2) / T_{1 / 2}\right)}{C_{\mathrm{Pha}}\left(1-e\left(-T_{\mathrm{TAT}} \ln (2) / T_{1 / 2}\right)\right)},
$$

where $\mathrm{CR}_{\mathrm{VOI}}$ is the counting rate derived from the reconstructed image and the segmented VOI, $C_{\mathrm{Pha}}$ is the known activity in the phantom, $T_{\mathrm{S}}$ is the starting time of the acquisition, $T_{\mathrm{CL}}$ is the activity calibration time, $T_{1 / 2}$ is the halflife of the radioisotope, and $T_{\mathrm{TAT}}$ is the total acquisition time of the study. To determine $S_{\text {SPECT }}$, the Jaszczak Standard SPECT Phantom ${ }^{\mathrm{TM}}$ was filled with a known and uniformly distributed solution of ${ }^{99 \mathrm{~m}} \mathrm{Tc}$. This experiment was carried out $(n=3)$ for activities of $37 \mathrm{MBq}, 185 \mathrm{MBq}$, and $370 \mathrm{MBq}$ (phantom concentrations of $0.005,0.026$, and $0.054 \mathrm{MBq} /$ $\mathrm{mL}$, respectively). $S_{\mathrm{SPECT}}$ was calculated with equation (5) and the VOIs were segmented in the reconstructed images $[20,23]$.

The correction factors $\left(\mathrm{CF}_{\mathrm{PVE}}\right)$ due to the partial volume effect (PVE) of the SPECT/CT system were calculated through a calibration method, in which five hollow spheres of different diameters were filled with equal ${ }^{99 \mathrm{~m}} \mathrm{Tc}$ activity concentrations $(0.818,0.409$, and $0.164 \mathrm{MBq} / \mathrm{mL})$ in a uniformly distributed background activity. This experiment was repeated for background ratios of $2: 1,5: 1$, and $10: 1$ $(n=3)$. The $\mathrm{CF}_{\mathrm{PVE}}$ for each sphere were calculated according to the following equation $[20,24]$ :

$$
\mathrm{CF}_{\text {PVE }}=\frac{A_{\text {Activimeter }}}{A_{\text {SPECT }}} \text {, }
$$

where $A_{\text {SPECT }}$ is the activity determined in the SPECT reconstructed image and $A_{\text {Act }}$ is the filling activity measured with the activimeter. For the mean $\mathrm{CF}_{\mathrm{PVE}}$ for each sphere, the size was calculated, and the obtained data was fitted in a function of the following equation:

$$
\mathrm{CF}_{\mathrm{PVE}}(\mathrm{VOI})=A e^{-a \mathrm{VOI}}+B e^{-b \mathrm{VOI}}+C e^{-c \mathrm{VOI}},
$$

where $A, B, C, a, b$, and $c$ are the fitting constants and VOI is the volume of interest in the sphere under study.

The activity in the VOIs $\left(A_{\mathrm{VOI}}\right)$ was calculated using the following equation:

$$
A_{\mathrm{VOI}}=\frac{R_{\mathrm{VOI}} \mathrm{CF}_{\mathrm{PVE}}}{S_{\mathrm{SPECT}}},
$$

where $R_{\mathrm{VOI}}$ is the counting rate in the VOI, $\mathrm{CF}_{\mathrm{PVE}}$ is the correction factor associated with the VOI, and $S_{\text {SPECT }}$ is the system sensitivity factor $[20,23]$. The counting rates of the SPECT images were obtained drawing ROIs in the SPECT/ CT slices of the VOI under study. All the SPECT reconstructions were decay-corrected.

(3) Hybrid Method. Considering that SPECT/CT quantification is more accurate, correction factors between imaging modalities were calculated (equation (9)) to scale the activity obtained from planar imaging:

$$
\mathrm{CF}_{\mathrm{Hyb}}=\frac{A_{\text {organ of interest in SPECT }}}{A_{\text {organ of interest in planar }}}
$$

where $\left(\mathrm{CF}_{\mathrm{Hyb}}\right)$ are the corrections factors of the hybrid method, $A_{\text {organ of interest in SPECT }}$ is the activity in the organ of interest quantified by SPECT, and $A_{\text {organ of interest in planar is }}$ the activity measured in the planar images [13].

$\mathrm{CF}_{\mathrm{Hyb}}$ were applied in the quantifications of the planar method $\left(A(t)_{\mathrm{P}}\right)$ to obtain the volumetric activity quantification $\left(A(t)_{\mathrm{VOI}}\right)$, according to the following equation: 


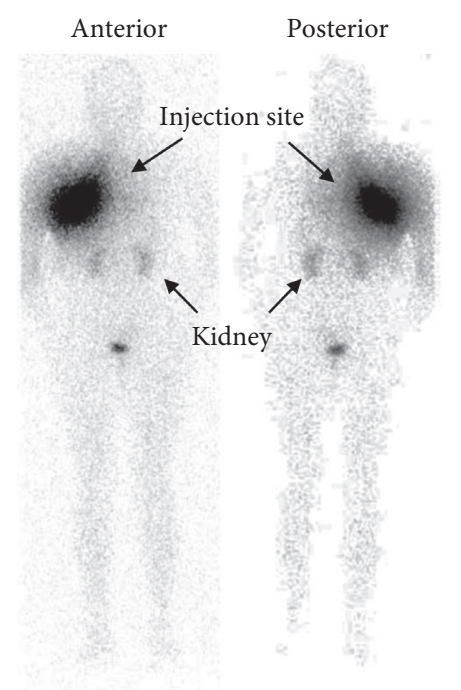

(a)

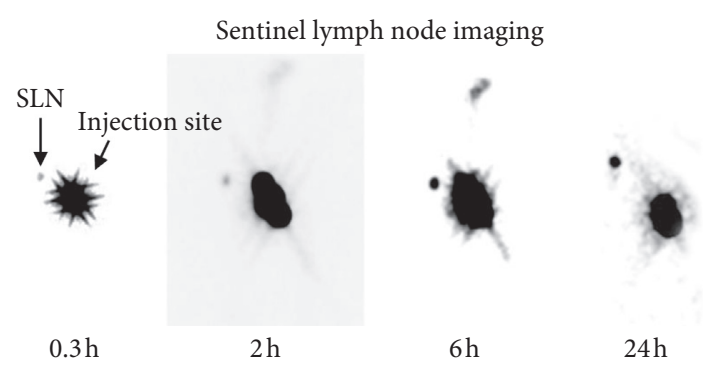

(b)

Figure 1: Planar images of the patient after the administration of ${ }^{99 \mathrm{~m}} \mathrm{Tc}-\mathrm{AuNP}$-mannose $(37 \mathrm{MBq})$. Anterior and posterior whole-body at $2 \mathrm{~h}$ after radiotracer administration (a) and frontal breast view at different times (b).

$$
A(t)_{\mathrm{VOI}}=\mathrm{CF}_{\mathrm{Hyb}} A(t)_{\mathrm{P}}
$$

The scaled \%INAs of each organ were fitted to threeexponential models using OLINDA/EXM.

\subsection{4. ${ }^{99 m} T c-A u N P-M a n n o s e ~ A b s o r b e d ~ D o s e ~ C a l c u l a t i o n s$.} The absorbed dose to organs was evaluated according to the following equation:

$$
D\left(r_{\mathrm{T}}, T_{\mathrm{D}}\right)=\sum_{r_{\mathrm{s}}} N\left(r_{\mathrm{s}}, T_{\mathrm{D}}\right) \mathrm{DF}\left(r_{\mathrm{T}} \longleftarrow r_{\mathrm{s}}\right)
$$

where $D\left(r_{\mathrm{T}}, T_{\mathrm{D}}\right)$ is the mean absorbed dose to a target tissue $r_{\mathrm{T}}$ from a source tissue $r_{\mathrm{s}}, N\left(r_{\mathrm{s}}, T_{\mathrm{D}}\right)$ is the total number of nuclear transformations that occurred in $r_{\mathrm{s}}$ over the doseintegration period $T_{\mathrm{D}}$, and $\mathrm{DF}\left(r_{\mathrm{T}} \longleftarrow r_{\mathrm{s}}\right)$ is the absorbed dose in $r_{\mathrm{T}}$ per nuclear transformation in $r_{\mathrm{s}}$. In this study, the equivalent absorbed dose estimates were obtained by entering the experimental $N\left(r_{s}, T_{\mathrm{D}}\right)$ values for all source organs into OLINDA/EXM $[19,25]$.

\section{Results and Discussion}

The SPECT detectors showed a linear response, as expected. $S_{\text {SPECT }}$ was $572.49 \mathrm{cps} / \mathrm{MBq}$. The $\mathrm{CF}_{\mathrm{PVE}}$ fitting is given by equation (12), in which the triexponential parametric analysis yielded a correlation coefficient of $R^{2}=0.99$ :

$$
\mathrm{CF}_{\mathrm{PVE}}(\mathrm{VOI})=1.09 e^{-0.38 \mathrm{VOI}}+1.11 e^{-2.68 \times 10^{-3} \mathrm{VOI}}+0.29 e^{-9.61 \times 10^{-5} \mathrm{VOI}} \text {. }
$$

Figure 1 shows the whole-body 2D images (left) and frontal 2D view of the injection site and sentinel lymph node (patient 1) acquired at different times. In this figure, only renal excretion is observed, mainly due to the radionanosystem functionalization with mannose $[5,16]$. Figures 2(a) and 2(b) display the frontal and lateral 3D images acquired at $6.5 \mathrm{~h}$ after the radio-nanosystem administration. Figure 2(c) illustrates a slice of the fused SPECT/CT imaging, where ${ }^{99 \mathrm{~m}}$ Tc-AuNP-mannose uptake in the SLN can be easily observed.

None of the 5 patients reported side effects such as chills, muscle cramps, decreased blood pressure, bradycardia, vomiting, coughing, itching, dyspnea, bronchospasm, flushing, nausea, hives, or dizziness after the radiolabeled nanoparticles were administered. The total number of nuclear transformations that occurred in the source organs (breast, SLN, urinary bladder, and kidneys) is shown in Table 2. The equivalent radiation absorbed doses and the effective dose of ${ }^{99} \mathrm{~m}$ Tc-AuNP-mannose are shown in Table 3.

The effective mean residence time $\left(\int_{t=0}^{t=\infty} A(t) \mathrm{d} t / A_{0}\right)$ of the nanoparticles in the SLN was calculated to be $0.92 \mathrm{~h}$, while the biological mean residence time (corrected by decay) was $6.13 \mathrm{~h}$. From the latter data, the safety of unlabeled AuNP-mannose could be questioned because of the possible biological damage that could be caused by the nanoparticle itself, associated with a prolonged AuNP-tissue interaction. In this regard, it is essential to mention that the effect of nanoparticles on cells and tissues change, depending on the type of interaction at the place of contact. Several trials have demonstrated that gold nanoparticles capped with citrate (from 5 to $13 \mathrm{~nm}$ ) caused an increase in the reactive oxygen species because AuNPs form strong Au-S bonds with intracellular glutathione and thiol-proteins $[26,27]$. However, in the case of AuNPs with mannose or peptides attached to their surface, the generation of reactive oxygen species is negligible, due to the biocompatibility and steric effect induced by the biomolecules, which circumvent Au-glutathione/Au-thiol-protein reactions [27].

In this study, absorbed dose calculations were assessed using hybrid (2D/3D) dosimetry under the assumption that planar imaging (2D) methods overestimate or underestimate 


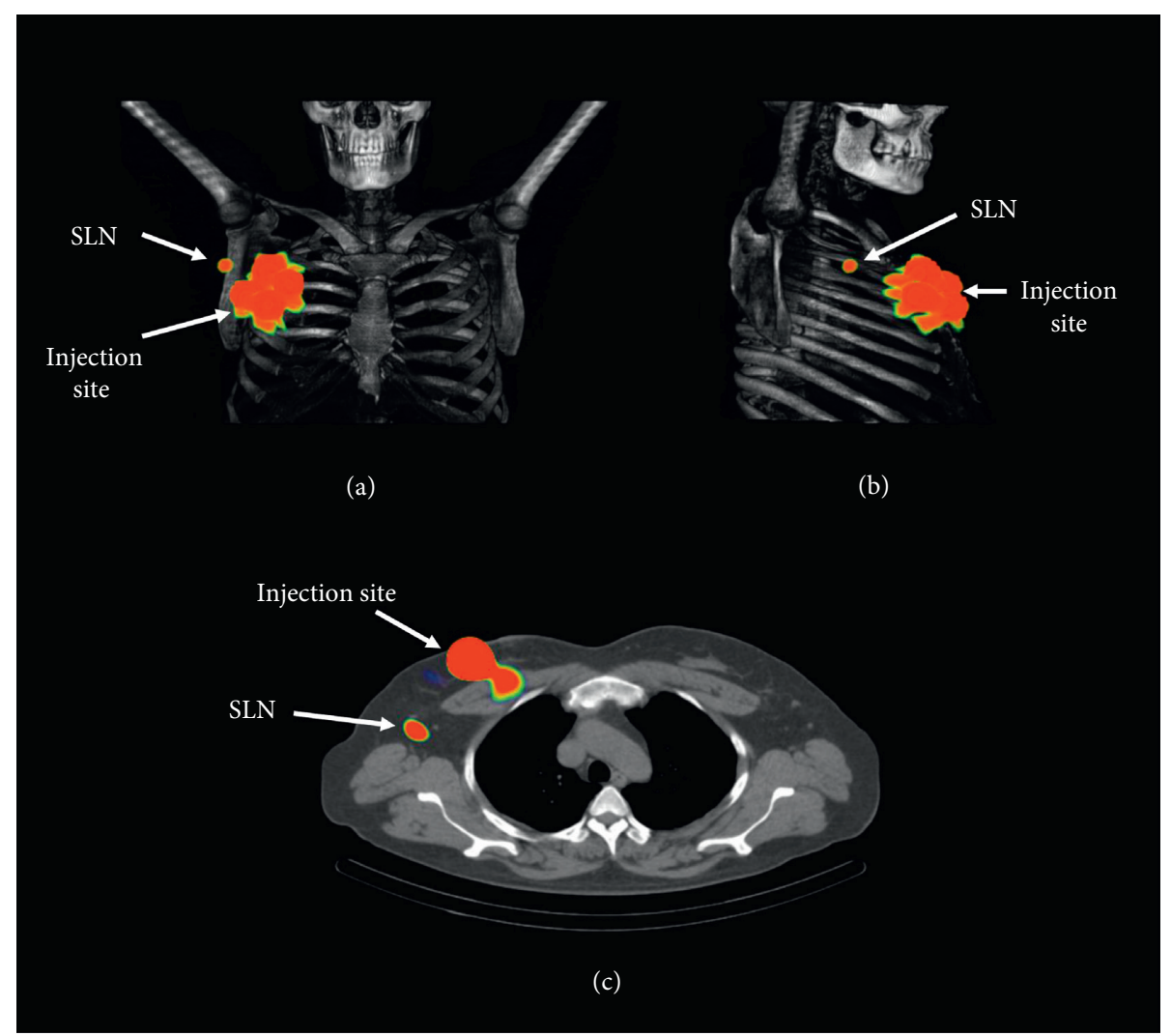

Figure 2: SPECT/CT imaging of the patient number 1 after $6.5 \mathrm{~h}$ of ${ }^{99 \mathrm{~m}} \mathrm{Tc}-\mathrm{AuNP}$-mannose $(37 \mathrm{MBq})$ administration. (a) Frontal and (b) lateral views. (c) Slice of the fused SPECT/CT imaging.

TABLE 2: Biokinetic model of ${ }^{99 m}$ Tc-AuNP-mannose calculated from five patients with breast cancer under an SLN protocol (37 MBq by intradermal periareolar administration in breast).

\begin{tabular}{|c|c|c|}
\hline Organ & Biokinetic model $A(t)_{\mathrm{VOI}}=\mathrm{CF}_{\mathrm{Hyb}} A(t)_{\mathrm{P}}$ & $N=\int_{t=0}^{t=\infty} A(t)_{\mathrm{VOI}} \mathrm{d} t(\mathrm{MBq} \cdot \mathrm{h} / \mathrm{MBq})($ mean $\pm \mathrm{SD})$ \\
\hline Breasts & $\begin{array}{c}A(t)_{\mathrm{VOI}}=71.80 e^{-0.23 t}+5.01 e^{-0.23 t}+2.27 e^{-0.23 t} \\
R^{2}=1\end{array}$ & $3.52 E+0 \pm 1.97 E-1$ \\
\hline Kidneys & $\begin{array}{c}A(t)_{\mathrm{VOI}}=0.74 e^{-5.22 t}+0.40 e^{-0.23 t}+0.12 e^{-0.12 t} \\
R^{2}=1\end{array}$ & $2.85 E-2 \pm 6.77 E-3$ \\
\hline Urinary bladder & $\begin{array}{c}A(t)_{\mathrm{VOI}}=8.88 e^{-12.32 t}+0.88 e^{-0.24 t}+1 \times 10^{-7} e^{-0.12 t} \\
R^{2}=1\end{array}$ & $4.25 E-2 \pm 1.58 E-2$ \\
\hline Sentinel lymph node & $\begin{array}{c}A(t)_{\mathrm{VOI}}=-66.50 e^{-0.23 t}+54.30 e^{-0.17 t}+12.203 e^{-0.23 t} \\
R^{2}=1\end{array}$ & $9.22 E-1 \pm 2.68 E-1$ \\
\hline Remainder of the body & $\begin{array}{c}A(t)_{\mathrm{VOI}}=21.30 e^{-0.44 t}+2.36 e^{-0.44 t}+0.12 e^{-0.44 t} \\
R^{2}=0.97\end{array}$ & $5.47 E-1 \pm 1.74 E-1$ \\
\hline
\end{tabular}

radiation absorbed doses due to tissue-activity overlapping or the location of small-size tissues $[13,15]$. Taking 3D SPECT dosimetry as a reference, Lehnert et al. [28] demonstrated that, in ${ }^{177} \mathrm{Lu}$-based therapies, the kidney absorbed dose is overestimated by $95 \%$ when $2 \mathrm{D}$ planar imaging is applied and reduced to $13 \%$ when hybrid $(2 \mathrm{D} / 3 \mathrm{D})$ dosimetry is used [28]. In another study, Koral et al. [13] observed an underestimation in the average tumor doses of small lesions in 12 patients under ${ }^{131}$ I-tositumomab therapy.

For comparative purposes, we also performed dosimetry calculations, eliminating 3D SPECT imaging data. In agreement with Lehnert et al. [28], a kidney radiation absorbed dose $45 \%$ higher $(0.11 \mathrm{mSv} / 37 \mathrm{MBq})$ than that obtained with the 2D/3D hybrid dosimetry $(0.08 \mathrm{mSv} /$ $37 \mathrm{MBq}$ ), was observed, which suggested a dose overestimation. A similar circumstance was observed for the breast and urinary bladder, where their radiation absorbed doses were overestimated by $5 \%(2 \mathrm{D}=5.58 \mathrm{mSv} / 37 \mathrm{MBq}, 2 \mathrm{D} /$ $3 \mathrm{D}=5.32 \mathrm{mSv} / 37 \mathrm{MBq})$ and $14 \%(2 \mathrm{D}=0.12 \mathrm{mSv} / 37 \mathrm{MBq}$, $2 \mathrm{D} / 3 \mathrm{D}=0.11 \mathrm{mSv} / 37 \mathrm{MBq})$, respectively.

In contrast to the aforementioned organs, the calculated radiation absorbed dose of the SLN using 2D-dosimetry was 1.77 -fold lower $(97.26 \mathrm{mSv} / 37 \mathrm{MBq})$ regarding the $2 \mathrm{D} / 3 \mathrm{D}$ hybrid dosimetry estimation ( $172.34 \mathrm{mSv} / 37 \mathrm{MBq})$. This SLN dose underestimation is mainly due to the limitations of planar imaging for the detection of small tissues, justifying 
TABle 3: Average equivalent and effective doses (mSv/MBq) of ${ }^{99 \mathrm{~m}} \mathrm{Tc}$-AuNP-mannose, calculated from five patients with breast cancer.

\begin{tabular}{lcc}
\hline Target organ & \multicolumn{2}{c}{ Equivalent doses (mean \pm SD) } \\
& Hybrid 2D/3D & $2 \mathrm{D}$ \\
\hline Adrenals & $1.28 E-03 \pm 1.60 E-04$ & $1.34 E-03 \pm 1.20 E-04$ \\
Brain & $3.46 E-04 \pm 9.85 E-05$ & $3.14 E-04 \pm 8.08 E-05$ \\
Breasts & $1.44 E-01 \pm 1.00 E-02$ & $1.51 E-01 \pm 8.67 E-03$ \\
Gallbladder wall & $1.06 E-03 \pm 1.61 E-04$ & $1.08 E-03 \pm 1.25 E-04$ \\
LLI wall & $5.84 E-04 \pm 1.25 E-04$ & $5.63 E-04 \pm 1.28 E-04$ \\
Small intestine & $5.99 E-04 \pm 1.27 E-04$ & $5.86 E-04 \pm 1.15 E-04$ \\
Stomach wall & $1.45 E-03 \pm 1.77 E-04$ & $1.48 E-03 \pm 1.24 E-04$ \\
ULI wall & $6.76 E-04 \pm 1.37 E-04$ & $6.63 E-04 \pm 1.18 E-04$ \\
Heart wall & $4.70 E-03 \pm 3.94 E-04$ & $4.88 E-03 \pm 2.96 E-04$ \\
Kidneys & $2.03 E-03 \pm 3.31 E-04$ & $3.02 E-03 \pm 7.98 E-04$ \\
Liver & $1.56 E-03 \pm 1.79 E-04$ & $1.60 E-03 \pm 1.27 E-04$ \\
Lungs & $3.61 E-03 \pm 3.13 E-04$ & $3.74 E-03 \pm 2.31 E-04$ \\
Muscle & $1.05 E-03 \pm 1.30 E-04$ & $1.06 E-03 \pm 9.70 E-05$ \\
Ovaries & $5.92 E-04 \pm 1.28 E-04$ & $5.71 E-04 \pm 1.30 E-04$ \\
Pancreas & $1.47 E-03 \pm 1.84 E-04$ & $1.51 E-03 \pm 1.33 E-04$ \\
Red marrow & $1.12 E-03 \pm 1.38 E-04$ & $1.14 E-03 \pm 9.83 E-05$ \\
Osteogenic cells & $2.22 E-03 \pm 3.59 E-04$ & $2.19 E-03 \pm 2.74 E-04$ \\
Skin & $1.38 E-03 \pm 1.33 E-04$ & $1.42 E-03 \pm 9.54 E-05$ \\
Spleen & $1.13 E-03 \pm 1.47 E-04$ & $1.18 E-03 \pm 1.08 E-04$ \\
Thymus & $4.46 E-03 \pm 3.70 E-04$ & $4.63 E-03 \pm 2.84 E-04$ \\
Thyroid & $8.18 E-04 \pm 1.25 E-04$ & $8.08 E-04 \pm 9.38 E-05$ \\
Urinary bladder & $2.88 E-03 \pm 8.72 E-04$ & $3.27 E-03 \pm 1.12 E-03$ \\
wall & $7.03 E-04 \pm 1.26 E-04$ & $7.04 E-04 \pm 1.61 E-04$ \\
Uterus & & \\
Sentinel lymph & $4.66 E+00 \pm 6.73 E-01$ & $2.63 E+00 \pm 1.04 E+0$ \\
node & & \\
Effective dose & $2.05 E-03 \pm 1.92 E-04$ & $2.12 E-03 \pm 1.38 E-04$ \\
(mSv/MBq) & & \\
\hline & &
\end{tabular}

the preference of 3D and SPECT/CT systems for its assessment [10-12].

Based on these results, it is considered that 2D/3D hybrid dosimetric calculations obtained in this research are more accurate than those assessed with the traditional 2D-conjugate-view method.

It is important to mention that the particle size of commercial ${ }^{99 \mathrm{~m}} \mathrm{Tc}$-colloids used for sentinel lymph node detection is also nanometric. The effective dose of colloidal rhenium sulfide (Nanocis, particle size $8-68 \mathrm{~nm}$ ) has been reported to be $4.7 \mu \mathrm{Sv} / \mathrm{MBq}$ and for ${ }^{99 \mathrm{~m}} \mathrm{Tc}-\mathrm{DTPA}$-mannosyl-dextran (Lymphoseek, particle size $\sim 7 \mathrm{~nm}$ ) $17.8 \mu \mathrm{Sv} / \mathrm{MBq}[29,30]$. However, the effective dose of ${ }^{99 \mathrm{~m}} \mathrm{Tc}$-AuNP-mannose nanoparticles $(20 \mathrm{~nm})$ was significantly lower $(2.1 \mu \mathrm{Sv} / \mathrm{MBq})$ regarding Nanocis and Lymphoseek. Radiolabeled gold nanoparticles also produce lower equivalent doses in the liver $(1.6 \mu \mathrm{Sv} / \mathrm{MBq})$ compared to Nanocis $(2.8 \mu \mathrm{Sv} / \mathrm{MBq})$ and Lymphoseek (1.8 $\mu \mathrm{Sv} / \mathrm{MBq})$. In kidneys, similar equivalent doses were observed between Nanocis $(1.8 \mu \mathrm{Sv} / \mathrm{MBq})$ and ${ }^{99 \mathrm{~m}} \mathrm{Tc}$-AuNP-mannose $(2.0 \mu \mathrm{Sv} / \mathrm{MBq})$ but they were different for Lymphoseek $(10 \mu \mathrm{Sv} / \mathrm{MBq})[29,30]$.

\section{Conclusions}

This is the first report in which radiolabeled gold nanoparticles are applied for molecular imaging in patients. This preliminary study suggests that the use of ${ }^{99 \mathrm{~m}} \mathrm{Tc}$-AuNPmannose for SLN detection in patients is safe. The effective dose calculated by hybrid dosimetry is at the level recommended for diagnostic studies $(<10 \mathrm{mSv})$.

The quantification processes based on $2 \mathrm{D}$ images tend to overestimate or underestimate the activity in regions and organs of interest, leading to inaccuracies at the time of the dosimetric calculations. Although these inaccuracies could be considered negligible during the assessment of diagnostic radiopharmaceuticals, in the case of therapeutic radiopharmaceuticals, treatment response of patients could be significantly affected.

\section{Data Availability}

The data used to support the findings of this study is included within the article.

\section{Conflicts of Interest}

The authors declare that there are no conflicts of interest regarding the publication of this paper.

\section{Acknowledgments}

This research was completed as part of the activities of the "Laboratorio Nacional de Investigación y Desarrollo de Radiofármacos, CONACyT-Mexico." This study was supported by the Mexican National Council of Science and Technology (CONACYT) (Grant: A1-S-36841).

\section{References}

[1] A. J. Wilhelm, G. Sophie Mijnhout, and E. J. F. Franssen, "Radiopharmaceuticals in sentinel lymph-node detection-an overview," European Journal of Nuclear Medicine, vol. 26, no. 13, pp. S36-S42, 1999.

[2] International Atomic Energy Agency, "Radiopharmaceuticals for sentinel lymph node detection: status and trends iaea radioisotopes and radiopharmaceuticals series publications," IAEA Radioisotopes and Radiopharmaceuticals Series, vol. 6, 2015.

[3] S. Vidal-Sicart and R. Valdés Olmos, "Sentinel node mapping for breast cancer: current situation," Journal of Oncology, vol. 2012, Article ID 361341, 7 pages, 2012.

[4] M. Auffan, J. Rose, J.-Y. Bottero, G. V. Lowry, J.-P. Jolivet, and M. R. Wiesner, "Towards a definition of inorganic nanoparticles from an environmental, health and safety perspective," Nature Nanotechnology, vol. 4, no. 10, pp. 634-641, 2009.

[5] B. E. Ocampo-García, F. D. M. Ramírez, G. Ferro-Flores et al., " $99 \mathrm{~m}$ Tc-labelled gold nanoparticles capped with HYNICpeptide/mannose for sentinel lymph node detection," Nuclear Medicine and Biology, vol. 38, no. 1, pp. 1-11, 2011.

[6] X. Zhang, "Gold nanoparticles: recent advances in the biomedical applications," Cell Biochemistry and Biophysics, vol. 72, no. 3, pp. 771-775, 2015.

[7] S. B. Lee, G. Yoon, S.-W. Lee et al., "Combined positron emission tomography and cerenkov luminescence imaging of sentinel lymph nodes using PEGylated radionuclide-embedded gold nanoparticles," Small, vol. 12, no. 35, pp. 4894-4901, 2016. 
[8] D. Dumani, I.-C. Sun, and S. Emelianov, "Detection of lymph node metastasis using photoacoustic imaging and glycolchitosan-coated gold nanoparticles," The Journal of the Acoustical Society of America, vol. 143, no. 3, p. 1930, 2018.

[9] H. W. Yeung, I. H. Cody, A. Turlakow et al., "Lymphoscintigraphy and sentinel node localization in breast cancer patients: a comparison between 1-day and 2-day protocols," The Journal of Nuclear Medicine, vol. 42, pp. 420-423, 2001.

[10] H. Lerman, U. Metser, G. Lievshitz, F. Sperber, S. Shneebaum, and E. Even-Sapir, "Lymphoscintigraphic sentinel node identification in patients with breast cancer: the role of SPECT-CT," European Journal of Nuclear Medicine and Molecular Imaging, vol. 33, no. 3, pp. 329-337, 2006.

[11] H. Lerman, G. Lievshitz, O. Zak, U. Metser, S. Schneebaum, and E. Even-Sapir, "Improved sentinel node identification by SPECT/CT in overweight patients with breast cancer," Journal of Nuclear Medicine: Official Publication, Society of Nuclear Medicine, vol. 48, no. 48, pp. 201-206, 2007.

[12] E. Even-Sapir, H. Lerman, G. Lievshitz et al., "Lymphoscintigraphy for sentinel node mapping using a hybrid SPECT/CT system," The Journal of Nuclear Medicine, vol. 44, pp. 1413-1420, 2003.

[13] K. F. Koral, Y. Dewaraja, J. Li et al., "Update on hybrid conjugate-view SPECT tumor dosimetry and response in 131I-tositumomab therapy of previously untreated lymphoma patients," The Journal of Nuclear Medicine, vol. 44, pp. 457464, 2003.

[14] M. Garkavij, M. Nickel, K. Sjögreen-Gleisner et al., "177Lu[DOTA0,Tyr3] octreotate therapy in patients with disseminated neuroendocrine tumors: analysis of dosimetry with impact on future therapeutic strategy," Cancer, vol. 116, no. S4, pp. 1084-1092, 2010.

[15] Y. K. Dewaraja, E. C. Frey, G. Sgouros et al., "MIRD pamphlet no. 23: quantitative SPECT for patient-specific 3-dimensional dosimetry in internal radionuclide therapy," The Journal of Nuclear Medicine, vol. 53, no. 8, pp. 1310-1325, 2012.

[16] B. Ocampo-García, G. Ferro-Flores, E. Morales-Avila, and F. D. M. Ramírez, "Kit for preparation of multimeric receptorspecific ${ }^{99 \mathrm{~m}}$ Tc-radiopharmaceuticals based on gold nanoparticles," Nuclear Medicine Communications, vol. 32, no. 11, pp. 1095-1104, 2011.

[17] C. E. Cox, S. Pendas, J. M. Cox et al., "Guidelines for sentinel node biopsy and lymphatic mapping of patients with breast cancer," Annals of Surgery, vol. 227, no. 5, pp. 645-653, 1998.

[18] C. L. Santos-cuevas, G. Ferro-flores, C. Arteaga, D. Murphy, and P. A. Pichardo-romero, "Targeted imaging of gastrinreleasing peptide receptors with ${ }^{99 \mathrm{~m}}$ Tc-EDDA/HYNIC[Lys3]-bombesin: biokinetics and dosimetry in women," Nuclear Medicine Communications, vol. 29, no. 8, pp. 741-747, 2008.

[19] C. Santos-cuevas, G. Ferro-flores, F. O. Garc et al., "177LuDOTA-HYNIC-Lys (Nal)-Urea-Glu: biokinetics, dosimetry, and evaluation in patients with advanced prostate cancer," Contrast Media \& Molecular Imaging, vol. 2018, Article ID 5247153, 10 pages, 2018.

[20] J. Zeintl, A. H. Vija, A. Yahil, J. Hornegger, and T. Kuwert, "Quantitative accuracy of clinical ${ }^{99 \mathrm{~m}} \mathrm{Tc}$ SPECT/CT using ordered-subset expectation maximization with 3-dimensional resolution recovery, attenuation, and scatter correction," Journal of Nuclear Medicine, vol. 51, no. 6, pp. 921-928, 2010.

[21] M. Cachovan, A. H. Vija, J. Hornegger, and T. Kuwert, "Quantification of ${ }^{99 \mathrm{~m}} \mathrm{Tc}-\mathrm{DPD}$ concentration in the lumbar spine with SPECT/CT," EJNMMI Research, vol. 3, no. 1, p. 45, 2013.
[22] J. A. Siegel, S. R. Thomas, J. B. Stubbs et al., "MIRD pamphlet no. 16: techniques for quantitative radiopharmaceutical biodistribution data acquisition and analysis for use in human radiation dose estimates," Journal of Nuclear Medicine: Official Publication, Society of Nuclear Medicine, vol. 40, no. 2, pp. 37S-61S, 1999.

[23] D. L. Bailey and K. P. Willowson, "Quantitative SPECT/CT: SPECT joins PET as a quantitative imaging modality," European Journal of Nuclear Medicine and Molecular Imaging, vol. 41, no. S1, pp. 17-25, 2014.

[24] S. Gnesin, P. L. Ferreira, J. Malterre, P. Laub, J. O. Prior, and F. R. Verdun, "Phantom validation of Tc-99m absolute quantification in a SPECT/CT commercial device," Computational and Mathematical Methods in Medicine, vol. 2016, Article ID 4360371, 6 pages, 2016.

[25] W. E. Bolch, K. F. Eckerman, G. Sgouros, and S. R. Thomas, "MIRD pamphlet no. 21: a generalized schema for radiopharmaceutical dosimetry-standardization of nomenclature," The Journal of Nuclear Medicine, vol. 50, no. 3, pp. 477-484, 2009.

[26] S. Tedesco, H. Doyle, J. Blasco, G. Redmond, and D. Sheehan, "Exposure of the blue mussel, Mytilus edulis, to gold nanoparticles and the pro-oxidant menadione," Comparative Biochemistry and Physiology Part C: Toxicology \& Pharmacology, vol. 151, no. 2, pp. 167-174, 2010.

[27] E. Morales-Avila, G. Ferro-Flores, B. E. Ocampo-García, and L. M. Gómez-Oliván, "Engineered multifunctional RGD-gold nanoparticles for the detection of tumour-specific," Journal of Biomedical Nanotechnology, vol. 8, no. 6, pp. 991-999, 2012.

[28] W. Lehnert, K. Schmidt, S. Kimiaei, T. Meyer, M. Bronzel, and A. Kluge, "Impact of modality (2D planar, 2D/3D hybrid, 3D SPECT) on kidneys absorbed dose in 177Lu-based PRRT," The Journal of Nuclear Medicine, vol. 59, p. 391, 2018.

[29] Nanocis, Summary of Product Characteristics, Irish Medicines Board, 2014, https://www.hpra.ie/img/uploaded/swedocuments/ LicenseSPC_PA0677-006-001_21032014130042.pdf.

[30] Lymphoseek, Highlights of Prescribing Information, FDA, Silver Spring, MD, USA, 2016, https://www.accessdata.fda. gov/drugsatfda_docs/label/2016/202207s005lbl.pdf. 